Case Report

\title{
FRIEDREICH ATAXIA - A CASE REPORT
}

\section{Deepthi R.V. ${ }^{1}$, Seema Pavanam ${ }^{2}$, Vijaya Shenoy ${ }^{3} \&$ Siddarth S. Joshi ${ }^{4}$}

${ }_{1,2}^{1,2}$ Assistant Professors, ${ }^{3}$ Professor \& HOD, ${ }^{4}$ Junior Resident, Department of Pediatrics, K.S. Hegde M edical Academy, Nitte University, Deralakatte, Mangalore - 575 018, Karnataka, India.

\author{
Correspondence \\ Deepthi R.V. \\ Assistant Professor, Department of Pediatrics, K.S. Hegde M edical Academy, Nitte University \\ Deralakatte, M angalore 5757018, Karnataka, India. E-mail : drdeepthirv@yahoo.com
}

\begin{abstract}
:
Friedreich ataxia is an autosomal recessive disorder, due to expansion of trinucleotide repeat in Frataxin gene which presents with ataxic gait, absent tendon reflexes, extensor plantar response and positive Romberg test. We present a child who came with complaints of progressive ataxia of gait since the age of 10 years and was diagnosed to have Friedreich ataxia. They have associated cardiomyopathy and endocrine abnormality like diabetes and hypothyroidism.
\end{abstract}

Keywords: Areflexia, Friedreich ataxia, Extensor plantar response, Romberg's sign, Trinucleotide repeat.

\section{Introduction:}

Friedreich ataxia (FA) an autosomal recessive disorder is the commonest of the inherited ataxias, affecting approximately 1-2 per 100,000 population ${ }^{1}$. It is due to homozygous expansion of a guanine adenine adenine (GAA) trinucleotide repeat in intron 1 of the Frataxin gene on chromosome 9. The usual presentation is progressive ataxia absent knee and ankle jerks and dysarthria. The natural history of this disease is one of relentless progression. The patient usually becomes wheelchair bound within 10-15 years of onset. Only a few reports have documented the onset of the disease during childhood and also due to rarity of the case, we are presenting a 11 year old child who presented with progressive ataxia of gait since the age of 10 years and on evaluation was diagnosed to have FA. Though FA presents with predominant neurological manifestations, they have associations with cardiomyopathy and endocrine abnormality, hence long term follow up is imperative.

\begin{tabular}{|c|}
\hline Access this article online \\
\hline Quick Response Code \\
\hline
\end{tabular}

\section{Case report:}

A 11 year old girl born of s e con d d e gre e consanguineous marriage presented with history of swaying while walking for past two years. There was associated difficulty in squatting and climbing down stairs. There was no history of tripping while walking, wasting of muscles tingling or paraesthesia in the lower limbs. Over one year it slowly progressed to gait instability. She did not have any feeding difficulties. There were no abnormalities in hearing or vision. There was no significant past or family history except for pes cavus in father.

Examination revealed high arched palate, abnormal dermatoglyphics, saddle gap in the feet, pes cavus (Fig 1) and kyphoscoliosis. Vitals were normal. She had microcephaly with subnormal intelligence. Cranial nerves and fundus were normal. She had wide based gait with ataxia and positive Romberg's sign. Child had dysmetria, dysdiadokokinesia and horizontal nystagmus. There was lower limb areflexia and extensor plantar response. There were no dysarthria, involuntary movements, calf muscle hypertrophy or fasciculations. Her sensory system was normal. MRI showed normal brain parenchyma and thinning of the cervical segment of the spinal cord (Fig 2). Nerve conduction velocities were normal. Electrocardiogram showed biventricular hypertrophy. There were no conduction blocks. Echocardiography thyroid profile and blood sugar were within normal limits. In view of cerebellar ataxia with bilateral pyramidal signs, loss of deep tendon reflexes, kyphoscoliosis and pes cavus a diagnosis of FA was made. Physiotherapy was initiated. 
She requires long term follow up for cardiomyopathy and diabetesmellitus.

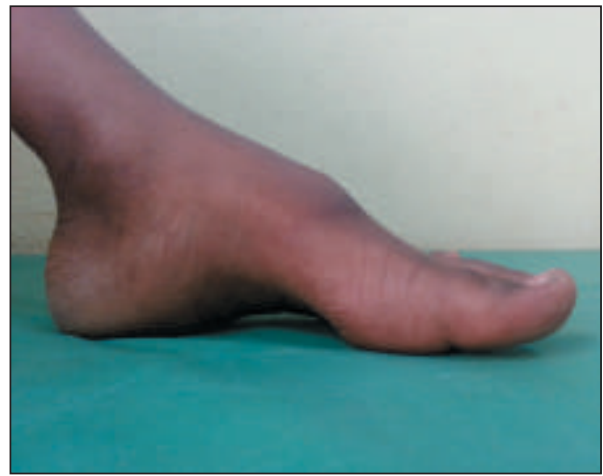

Fig. 1 Pes Cavus

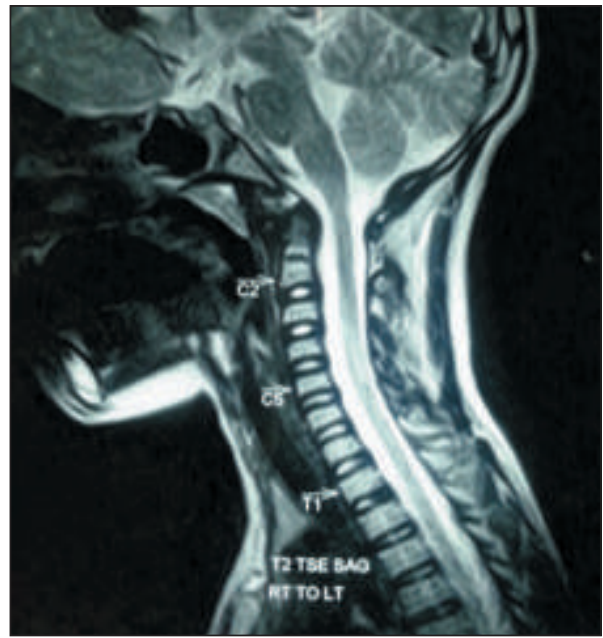

Fig. 2 Thinning of Cervical Segment of Spinal Cord

\section{Discussion:}

FA affects central and peripheral nervous systems, heart, skeleton and endocrine pancreas. The estimated carrier frequency ranges from 1:50 to 1:100 in people of European, North African, Middle Eastern and Indian origin. The point prevalence is 3 per one lakh population. ${ }^{1} \mathrm{FA}$ is typically a disease of young adults with no gender predilection. The varied initial diagnosis and the delay in reaching a final diagnosis reflect the lack of knowledge and published reports on FA in children. A family history of Friedreich's ataxia may lead to early diagnosis. M ean age of onset is $10.52 \pm 7.4$ years and the average age at death was 37 years.

The following neurological signs are most frequent: Gait ataxia, dysmetria of arms and legs, dysarthria, head titubation, atrophy and weakness of the distal extremities, absence of muscle stretch reflexes, Babinski sign, loss of joint and vibratory senses and superimposed glove and stocking type sensory neuropathy. ${ }^{2}$ Gait ataxia is the earliest symptom in the vast majority. Within five years of symptom onset, most individuals with FA exhibit scanning dysarthria, lower extremity weakness, and diminished or absent joint position and vibration sense. Involvement of peripheral sensory and motor neurons results in a mixed axonal neuropathy. Muscle weakness is most prominent in hip extensors and abductors. Spasticity in the lower limb is common and can be significant, affecting foot plantar flexors and inverters to a greater extent than dorsiflexors and everters.

Neurologic manifestations result from progressive degeneration of the dorsal root ganglia, posterior columns, corticospinal tracts, the dorsal spinocerebellar tracts of the spinal cord, and cerebellum.

Optic nerve atrophy, often asymptomatic, occurs in approximately $25 \%$ of individuals with FA. Cognition is generally not impaired in FA but motor and mental reaction times can be significantly slowed. Complex oculomotor disturbances are characteristic and include abnormal saccades and square wave jerks. Despite normal pure-tone audiometry, patients with FA may have problems with speech perception which is suitably termed as "auditory neuropathy/dyssynchrony". There is also evidence of vestibular dysfunction.

Scoliosis and pes cavus are extremely common in FA (60-79\%) and is clearly progressive. Diabetes mellitus occurs in $8-32 \%$ of FA patients. Progressive systolic dysfunction is common. Cardiomyopathy was the immediate or contributory cause of death in $83.3 \%$. $^{3}$ Subjective symptoms of exertional dyspnea, palpitations and anginal pain may be present in moderately advanced disease. Arrhythmias and congestive heart failure are the most common cause of mortality. M ural thrombi in the left ventricle are common and some patients with FA have embolic strokes.

The gene for FA was mapped to chromosome 9ql3 in 1988 
by Chamberlain and colleagues. An inverse relation was found between the size of the smaller and both age at onset and time until confinement to a wheelchair. The association of cardiomyopathy with large expansion is important for prognosis. ${ }^{4}$ The incidence and progression of cardiomyopathy is directly proportional to the length GAA expansion. Genetic confirmation requires demonstration of triple repeat sequences of GAA on chromosome 9 .

According to Harding's criteria an age of onset before 25 years, progressive ataxia, absent deep tendon reflexes and dysarthria are considered to be essential criteria. Scoliosis, pyramidal weakness in lower limbs, absent tendon reflexes in arms, impairment of vibration and joint position sense and cardiomyopathy are additional criteria. ${ }^{5}$ Nystagmus, optic atrophy, deafness, distal amyotrophy, pes cavus and diabetes mellitus are other criteria found in less than $50 \%$ cases.

\section{References}

1. Koeppen AH. Friedreich's ataxia: Pathology, pathogenesis and molecular genetics. J Neurol Sci.2011; 303:1-29.

2. Delatycki MB, Corben LA. Clinical features of Friedreich ataxia. J Child Neurology 2012; 27(9): 1133-1137.

3. Payne RM, Wagner GR. Cardiomyopathy in Friedreich ataxia: Clinical findings and Research. J. Child Neurology 2012; 27(9):1179-86.

4. Filla A, De Michele G, Cavalcanti F, Pianese L, M onticelli A, Campanella G.The relationship between trinucleotide (GAA) repeat length and clinical features in Friedreich's ataxia. Am J Hum Genet 1996; 59(3):559-560.

5. Harding AE. Friedreich's ataxia: a clinical and genetic study of 90 families with an analysis of early diagnostic criteria and intrafamilial clustering of clinical features. Brain 1981; 104:589-620.

6. Durr A, Cossee M, Agid Y, Campuzano V, M ignard C, Penet C, M andel IL, Brice A, Koenig M. Clinical and genetic abnormalities in patients with Friedreich's ataxia. N EnglJ M ed. 1996; 335: 1169-75.

7. Bhidyasiri RB, Perlman SL, Stefan M P, Daniel HG. Late onset Friedreich ataxia. Phenotypic analysis, MRI findings and review of literature. Arch Neurology 2005; 62(12): 1865-1869.

8. Kearny M, Ovrell RW, Fahey M, Pandolfo M. A ntioxidants and other pharmacological treatment for Friedreich ataxia. Cochrane Database Systematic Review 2012 April 18; 4: CD007791.
Abnormalities in nerve conduction studies are severely slowed or absent sensory conduction with normal motor conduction velocity, motor nerve conduction velocity of greater than $40 \mathrm{~m} / \mathrm{s}$ with reduced or absent sensory nerve action potential and absent $\mathrm{H}$ reflex. ECG is abnormal in the vast majority with $\mathrm{T}$ wave inversion, left axis deviation, and repolarization. Echocardiographic evaluation may reveal left ventricular hypertrophy that is more commonly asymmetric than concentric. ${ }^{6}$

MRI brain shows thinning of the cervical spinal cord and atrophy of the superior cerebellar peduncles. ${ }^{7}$ Reduced Nacetyl aspartate in the cerebellum has been demonstrated by Proton Magnetic Resonance Spectroscopy. In our patient the clinical signs agree with those of Harding: ataxic gait, absent tendon reflexes, extensor plantar response and positive Romberg test with additional scoliosis and pes caves. Confirmation of genetic diagnosis was not possible in our case due to financial constraints of the patient. 